\title{
An Intrusion-Resilient Authorization and Authentication Framework for Grid Computing Infrastructure
}

\author{
Yuanbo Guo ${ }^{1,2}$, Jianfeng $\mathrm{Ma}^{2}$, and Yadi Wang ${ }^{1}$ \\ ${ }^{1}$ School of Electronic Technology, Information Engineering, \\ University, Zhengzhou, Henan 450004, China \\ yuanbo_g@hotmail.com \\ 2 The Ministry of Education Key Laboratory of Computer Networks and \\ Information Security, Xidian University, Xi'an, 710071, China
}

\begin{abstract}
A correctly and continually working authorization and authentication service is essential for the grid computing system, so it is very necessary to maintain efficient this service with high availability and integrity in the face of a variety of attacks. An intrusion-resilient framework of authorization and authentication service for grid computing system is presented in this paper. This service is able to provide fault tolerance and security even in the presence of a fraction of corrupted authorization and authentication servers, avoiding any single point of failure. We use a cryptographic $(f, n)$ secret sharing scheme to distribute parts of the clients' proxy certificates and use a secure multi-party computation scheme to perform the signatures such that the proxy certificate can be issued in a distributed fashion without reassembly when a legal client registrant at the Globus host. By using Non-Malleable Proof, the "man-in-the-middle attack" can be prevented; by distributing the secret data across several authorization and authentication servers, the compromise of a few servers will not compromise the availability of data. And, under the assumption of a Diffie-Hellman decisional problem, a passive adversary gets zero knowledge about the system's private key $X$, and so cannot to issue the certification for any client, neither to impersonate a legal authorization and authentication server.
\end{abstract}

\section{Introduction}

At the base of any grid environment, there must be mechanisms to provide security, including authentication, authorization, data encryption, and so on. The Grid Security Infrastructure (GSI) component of the Globus Toolkit provides robust security mechanisms. Especially, it provides a single sign-on mechanism, so that once a client is authenticated, the certificate authority in the GSI will generate a proxy certificate and send it to the client, then the client can use the proxy certificate to perform actions within the grid computing system. But this mechanism has its own drawback. For example, since all clients' certificates are maintained by the certificate authority, it requires the certificate authority to be trusted and so introduces dependence upon the certificate authority and thus creates a single point of failure, and, if successfully compromised, would immediately lead to the exposure of all clients' confidentiality of certificates, interruption communication, or other forms of denial of service. 
To address this issue, in this paper we present an intrusion-resilient authorization and authentication framework for grid computing infrastructure, that removes this single point of failure by distributing the authorization and authentication service among $n$ authorization and authentication servers (which will be denoted as AASs briefly in the remaining of the paper). The framework uses a verifiable secret sharing scheme to distribute shares of the system's secret to AASs and use a secure multiparty computation scheme to perform the signatures such that the proxy certificate can be signed in a distributed fashion without reassembly. When a legal client want to sign-on the grid computing system, he/she sends a sign-on request to the Globus host firstly, the Globus host will forward this request to a his-own-choosing subset of the $n$ $A A S s$, and the contacted $A A S s$ answer with some information enabling the client to compute his own proxy certificate and verify its correctness.

Our framework is intended to tolerate the compromise of up to $f A A S s$, where $3 f+1 \leq n$. Compromised $A A S s$ are assumed to be under the full control of an attacker and to have Byzantine behavior ${ }^{1}$, but it is also assumed that the attacker cannot break the cryptographic algorithms used. Under these assumptions, our service ensures the confidentiality, integrity and availability of authorization and authentication service for grid computing infrastructure. Also, our system is designed to be easy to embed into existing grid computing environment. For example, the AASs are built with commodity PCs, an administrator can just swap a failed host out with another.

\section{System Architecture}

Let Client $=\left\{C_{1}, \ldots, C_{m}\right\}$ be a set of $m$ clients and let $A A S=\left\{A A S_{1}, \ldots, A A S_{n}\right\}$ be a set of $n A A S \mathrm{~s}$ (authorization and authentication servers for grid computing infrastructure). All of them share a global clock (i.e., the system is synchronous). Each client connects himself or herself with $2 f+1 A A S$ s by point-to-point encrypted and authenticated channels, where $f$ is the threshold of secret sharing scheme we used. The member in the $A A S$ s can be good (i.e., it honestly executes the protocol) or bad (i.e., it is controlled by an adversary and can deviate from the protocol in arbitrary ways), but a majority of good $A A S$ s are always present across the system.

The service works as follows. A dealer, which is needed only in the system initialization phase, distributes system's private key $X$ among all $n A A S$ s by using Feldman's $(f+1, n)$ VSS scheme [3] straight forward. So along with the secret shares, he also generates a verification share to go along with each key share, and broadcast the verification shares to all clients.

Each secret share is then distributed secretly to a different $A A S$, and all corresponding verification shares are broadcast to all of the clients. No $A A S$ knows the whole $X$, but only knows his own assigned share.

The architecture of our intrusion-resilient authorization and authentication service can be illustrated as Figure 1.

\footnotetext{
${ }^{1}$ A compromised host deviates arbitrarily from its specified protocols. It is also called arbitrary behavior or malicious behavior.
} 
Each $A A S$ maintains a configuration file which specifies what types of resource are allowed to be access by which client. When a client wants to apply for some services of or execute a task on the grid computing system, he/she registers himself/herself at the Globus hosts firstly, with the submission of his/her password or some others type of credential and the requested services or tasks. Then, Globus host will performs the initial validation; if success, it sends an authentication and authorization request to the $A A S$ s on behalf of the client. Based on the identification of the client, each $A A S$ consults its configuration files to determine what resources in the grid environment are authorized to the client, and then uses its share of the system's private key to compute and send a share of proxy certificate to the client, which we called sub-certificate in the remaining paper. It also uses its verification share to compute a zero-knowledge proof which will prove the correctness of its sub-certificate.

These sub-certificate and zero-knowledge proofs are then collected by the Globus host. Then, the host chooses $f$ of them. After verifying the correctness of all of the proofs of these sub-certificates, it can combine them to form the actual proxy certificate and send it to the client. Afterwards, the client can request the needed resources to the grid computing system by using this proxy certificate.

\section{Resources of the Grid Computing System}

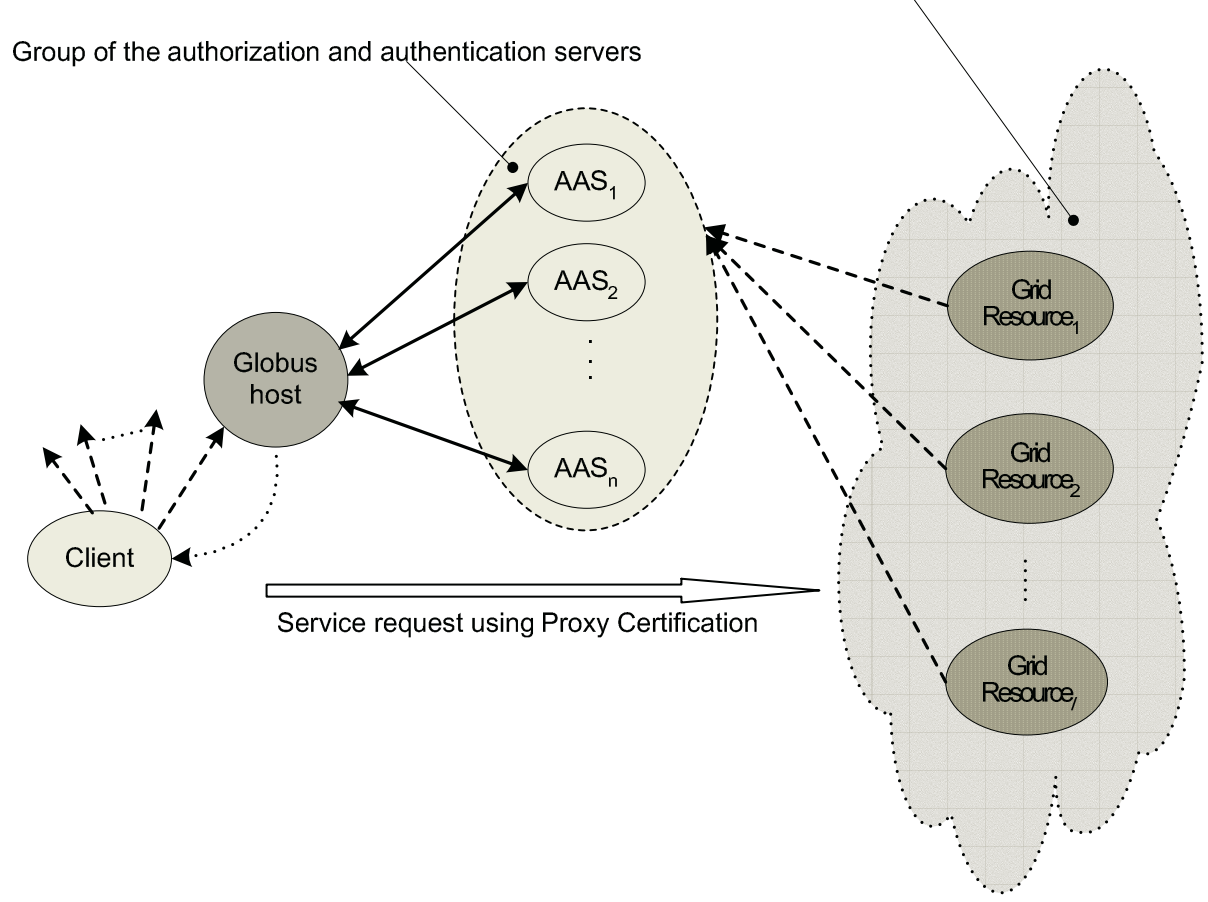

Fig. 1. Architecture of intrusion-resilient authorization and authentication service for grid computing environment 


\section{The Details of the Framework}

Denote the system's private key as $X_{A A S}$ and the corresponding public key $Y_{A A S}$,. Denote a client $C^{\prime}$ 's $X_{C}$ and $Y_{C}$. Our scheme starts with a large prime $p=2 q+1$, where $q$ is also a large prime, and a generator $g$ for the subgroup $G_{q}=\left\{i^{2} \mid i \in Z_{p}^{*}\right\}$ of quadratic residues of $Z_{p}^{*}$. A collision-free one-way hash function $H(x)$ is also needs to be made public. Then, our framework can be divided into 3 phase:

Initialization phase: The dealer distribute the system's private key $X_{A A S} \in \mathrm{Z}_{q}^{*}$ among all $A A S$ by using Feldman's $(f+1, n)$ VSS scheme [3] straight forward.

1) Select randomly $f$ elements $a_{l}, \ldots, a_{f}$ of $Z_{q}^{*}$. These coefficients define a polynomial of degree $\mathrm{t}$ in $\mathrm{Z}_{q}^{*}[X]$ :

$$
F(X)=X_{A A S}+a_{1} X+a_{2} X^{2}+\ldots .+a_{f} X^{f}
$$

2) Compute $X_{1}, \ldots, X_{\mathrm{n}}$ of $Z_{q}$ and $g_{1}, \ldots, g_{n}$ of $Z_{p}$ as follows:

$$
X_{i}=F(i) \bmod q \text { and } g_{i}=g^{X_{i}} \bmod p
$$

The shares $X_{1}, \ldots, X_{\mathrm{n}}$ must then be distributed secretly to the $n$ authorization and authentication servers $A A S_{1}, \ldots, A A S_{n}$, respectively. The generator $g$ and the elements $g_{1}, \ldots, g_{n}$ are made public. They must be known by all clients and all $A A S \mathrm{~s}$. Each $A A S_{i}$ can verifies the share $X_{\mathrm{i}}$ he or she received by using $g$ and $g_{i}$.

As in Shamir's secret sharing scheme ${ }^{[4]}$, any subset of $f+1$ values among $X_{1}, \ldots, X_{\mathrm{n}}$ allows one to reconstruct $F$ by interpolation, and then to compute the value $X_{A A S}=$ $F(0)$. For example, given $X_{i_{1}}, \ldots, X_{i_{f+1}}$, one has $X_{A A S}=\sum_{s=1}^{f+1} b_{i_{s}} X_{i_{s}} \bmod \mathrm{q}$, where $b_{i_{s}}=\prod_{j=1, j \neq s}^{f+1} \frac{\mathrm{i}_{\mathrm{j}}}{\mathrm{i}_{\mathrm{s}}-\mathrm{i}_{\mathrm{j}}} \bmod q$

Authorization Phase: The grid computing system authorizes AASs to authenticate the clients and issue the corresponding proxy certificates. Each grid resources $G R_{i}$, where $\mathrm{i}=1,2, \ldots, l$, is associated with an access control table $C L_{i}$, which is used to specifying what types of access to this resources of which client are allowed. After authorization, all $G R_{i}$ 's access control tables are maintained by each $A A S$.

Registration and Proxy Certificate Generation Phase: When a client $C$, whose ID is $I D_{C}$, wants to apply for some services or execute some tasks, he/she registers at the Globus host firstly. The client should submit to Globus host his/her password or other credential and the requested services or tasks. On receiving these information, the Globus host will then performs the initial validation of the client; if successes, it sends an authentication and authorization request to a robust subset of $A A S s$ (in our case, the servers of size at least $2 f+1$, where $f$ is the threshold of the secure secret sharing scheme we used) on behalf of the client. Each $A A S$ does the following: 
1) Compute sub-signature of client $C$ 's private key $S C_{i}=X_{C}^{X_{i}} \bmod p$;

2) Randomly select $r \in Z_{q}^{*}$ and compute

$$
u=g^{r} \bmod p \text { and } v=X_{C}{ }^{r} \bmod p
$$

3) Compute $s_{i}=H\left(g, g_{i}, I D_{C}, S C_{i}, u, v\right)$ and $t_{i}=r+s_{i} . X_{i} \bmod q$.

4) Based on the identification of the client, each $A A S$ consults its configuration files to determine what resources in the grid environment are authorized to the client, and then generate a sub-certificate $\left(S C_{i}, s_{i}, t_{i}, C\right)$ for client $C$ (denoted as $\operatorname{Cert}_{C}^{i}$ ), and then send it to the Globus host.

In step 3) and 4), both $s_{i}$ and $t_{i}$ are used here to prove the validity of $S C_{i}$ without revealing $X_{i}$. This uses a technique of non-malleable proof proposed by [5].

After receiving $\operatorname{Cert}_{C}^{i}$ send by $S_{i}$, the Globus host does following:

1) Compute $u^{\prime}=g^{t_{i}} / g_{i}^{S_{i}} \bmod p$ and $v^{\prime}=X_{C}^{t_{i}} / S C_{i}^{S_{i}} \bmod p$

2) compute $s_{i}{ }^{\prime}=H\left(g, g_{i}, I D_{C}, S C_{i}, u^{\prime}, v^{\prime}\right)$

If $s_{i}{ }^{\prime}=s_{i}$, then the client accept the proof and get the sub-certificate $\operatorname{Cert}_{C}^{i}$; else, Globus host can raise an alarm to the administrator of system.

After receiving and checking $f+1$ sub-certificates $\operatorname{Cert}_{C}^{i}, \ldots, \mathrm{Cert}_{C}^{i+f+1}$ from different $f+1 A A S s$, the Globus host compute $S C=\prod_{s=1}^{f+1} S C_{i_{s}}^{b_{i_{s}}} \bmod p$, where $b_{i_{s}}$ is obtained from $S_{i_{1}}, \ldots, S_{i_{f+1}}$ and $b_{i_{s}}=\prod_{j=1, j \neq s}^{f+1} \frac{i_{j}}{i_{s}-i_{j}} \bmod q$. By this interpolation method, Globus host can obtain $S C=X_{C}^{X} \bmod p$.

Then, a proxy certificate containing the signature $S C$ for client $C$ can be generated and send to client $C$ by Globus host.

Afterwards, the proxy certificate can be used by the client to access the grid resources he/she needs.

\section{Security Analysis}

\subsection{Correctness and Robustness}

By correctness and robustness, we mean that all legal clients can get a correct proxy certificate if there are no more than $f$ dishonest AASs.

Theorem 1: If there are no more than $f$ dishonest $A A S s$, then all legal clients can get a correct proxy certificate.

Proof: In our case, we only need to show that Globus host will compute a correct $S C=X_{C}^{X}$ for legal client $C$.

From the details of the framework we know that, before Globus host compute and recover $S C$, it has received and verified $f+1$ sub-certificates $\operatorname{Cert}_{C}^{i}, \ldots, \operatorname{Cert}_{C}^{i+f+1}$ 
from different $f+1 A A S s$. Then by using interpolation method, Globus host can compute $S C=\prod_{s=1}^{f+1} S C_{i_{s}}^{b_{i_{s}}}=\prod_{s=1}^{f+1}\left(X_{C}^{X_{i}}\right)^{b_{i_{s}}}=\left(X_{C}^{X_{i}}\right)^{\sum_{s=1}^{f+1} b_{i_{s}} X_{i_{s}}}=X_{C}^{X}$.

\subsection{Security Against Impersonators Attack}

Impersonations means that server $A A S_{j}$ impersonate server $A A S_{i}$ to send message $\left(S C_{i}\right.$, $\left.s_{i}, t_{i}, C\right)$ to the Globus host.

Theorem 2: In the random oracle model, a dishonest $A A S$ cannot forge, with a non negligible probability, a valid proof for a valid sub-certificate.

Proof: From the above scheme we know that $S C_{i}, s_{i}, t_{i}$ satisfy the following equations:

$$
\begin{aligned}
& g^{t_{i}}=g^{r+s_{i} \cdot X_{i}}=g^{r} \cdot\left(g^{X_{i}}\right)^{s_{i}}=u \cdot g_{i}^{s_{i}} \\
& X_{C}^{{ }^{t_{i}}}=X_{C}{ }^{r+s_{i} \cdot X_{i}}=X_{C}{ }^{r} \cdot\left(X_{C}{ }^{X_{i}}\right)^{s_{i}}=v \cdot S C_{i}^{s_{i}}
\end{aligned}
$$

Since $g^{t_{i}}$ and $g_{i}^{s_{i}}$ are both in $G$, by the closure of $G$ under multiplication we know that Equation (4.1) yields $u \in G$. This means that it exists $\lambda$ such that $u=g^{\lambda}$. So we have $g^{t_{i}}=g^{\lambda} \cdot g_{i}^{s_{i}}=g^{\lambda} \cdot g^{X_{i} s_{i}}$, which implies: $t_{i}=\lambda+s_{i} \cdot X_{i}$. Now Equation (4.2) becomes:

$$
X_{C}^{t_{i}}=v S C_{i}^{s_{i}} \Leftrightarrow X_{C}^{\lambda+S_{i} \cdot X_{i}}=v S C_{i}^{s_{i}} \Leftrightarrow X_{C}^{\lambda} v^{-1}=\left(X_{C}{ }^{-X_{i}} \cdot S C_{i}\right)^{s_{i}}
$$

This yields two possible cases:

(1) $S C_{i}=X_{C}{ }^{X_{i}}$. In this case, the sub-certificate is correct. $v=X_{C}{ }^{\lambda}$ and for all $s_{i} \in Z_{q}$, the verifier equations trivially hold.

(2) $S C_{i} \neq X_{C}{ }^{X_{i}}$. In this case, we must have

$$
s_{i}=\log _{X_{C}-x_{i} \cdot S C_{i}} X_{C}^{\lambda} v^{-1}
$$

From equation (4.3), one can see that, if $S C_{i}$ is not a valid sub-certificate, once the triplet $\left(S C_{i}, u, v\right)$ is chosen, then there exists a unique $s_{i} \in Z_{q}$ that satisfies the verifier equations. In the random oracle model, the hash function $H$ is assumed to be perfectly random. Therefore, the probability that $H\left(g, g_{i}, I D_{C}, S C_{i}, u, v\right)$ equals $s_{i}$ is $\frac{1}{q}$, once $\left(S C_{i}, u, v\right)$ fixed.

On the other hand, if the attacker perform an adaptively chosen message attack by querying an oracle $N$ times, the probability for the attacker to find a triplet $\left(S C_{i}, u, v\right)$, such that $H\left(g, g_{i}, I D_{C}, S C_{i}, u, v\right)$ is $P_{\text {success }}=1-\left(1-\frac{1}{q}\right)^{N} \approx \frac{N}{q}$, for large $q$ and $N$. Now if $k$ is the number of bits in the binary representation of $q$, then $P_{\text {success }} \leq \frac{N}{2^{k}}$. Since a computationally bounded server can only try a polynomial number of triplets, then when $k$ is large, the probability of success, which is $P_{\text {success }} \leq \frac{N}{2^{k}}<<1$, is negligible. 


\subsection{Security Against Secret Compromise}

The most serious attack is for an attacker to compromise $f A A S s$ and reconstruct the system's private key $X$, and then to issue the certification for any client, whether legal or illegal. To combat this, $f$ should be chosen to be sufficiently large to make compromising $f$ machines exceedingly difficult.

Arbitrarily setting $f$ to be very large, however, has reliability concerns. In the extreme case, if $f=n$, then the system has $n$ points of failure-where a proxy certificate cannot be issued. $f$ must be chosen so that $n-f-1$ is sufficiently large to handle node failures.

Secondly, each machine will, ideally, be running a different operating system with different versions of software to make compromising a machine more difficult.

\subsection{Security Against Denial of Service}

Since $f$ machines are necessary to successfully compute a correct proxy certificate for the legal client and there are $n$ machines in total, if $n-f-1$ of them crashes or fails, the key will not be able to be computed. To prevent attackers from compromising or flooding $n-f$ - 1 machines, $n$ and $f$ will need to be appropriately chosen to make this probabilistically low. In the case where machines crash because they are faulty, we can bound the probability of failure.

\subsection{Failure Handling}

Consider that each $A A S$ fails—crashes or does not respond-with probability $r$. We assume $r$ is independent. The probability that a key cannot be computed is:

$$
p_{f}=r^{f}
$$

That is, $p_{f}$ is the probability that the system is unable to perform useful work; a state of failure.

Solving for $r$, we find: $\quad r=p_{f}{ }^{1 / f}$

Obviously, any system will need to set $n$ and $f$ such to maximize $r$.

\section{Discussions and Conclusion}

A combination of fairly standard secure multi-party computation algorithm, secret sharing scheme, zero knowledge proof and traditional authorization protocols was used to develop an intrusion-resilient framework of authorization and authentication for grid computing environment, which can provide correct services even in the presence of faulty insiders of $A A S s$. Its security requirements can be shown to theoretically hold if no more than $f A A S \mathrm{~s}$ are compromised, the attacker does not break the cryptographic algorithms, and the network assumptions are satisfied. But it is obviously that the claim of no more than $f A A S$ s are compromised relies on the assumption that it is substantially harder for an attacker to penetrate several $A A S$ s than a single one. In fact, with enough time and resources, a determined attacker can compromise a sufficient number of $A A S \mathrm{~s}$ to break the system. To justify this assumption we rely on 
two complementary approaches: diversity and proactive updates. With diversity, an attacker has to devise different penetration mechanisms for each server, so that it is impossible for him/her to work to kill other $A A S \mathrm{~s}$ at once when he/she kills one. Diversity can be achieved by running a different version of our software on each of the $A A S \mathrm{~s}$, running on different hardware and operating systems, and putting the different $A A S$ s under the responsibility of different administrator.

Proactive security is the ability to gracefully recover from compromises in such a way that any past compromises don't assist a current attacker. Proactive update implies that once every time period (say once an hour) the $A A S$ s compute a new sharing of the same system's privacy key. Consequently, not only does the attacker have to penetrate multiple $A A S \mathrm{~s}$, he/she has to do so within a single time period. Proactive update is normally implemented by re-sharing the private key in such a way that the new shares have no relationship to the old shares, and therefore if an attacker compromises some $A A S s$, these $A A S s$ are recovered, and then he/she compromises more $A A S s$, the information he gains isn't enough to compute the key.

However, due to the organization's budget and security constraints, the parameters of the system's diversity and proactive should be set accordingly.

Finally, it should be noted that, even with above measures, resilience is not absolute in the real world. Our scheme can also be vulnerable to network-based denial-ofservice attack based on flooding, though this attack is not specific to our system, as current TCP/IP protocols make it difficult to defend against such attacks in any system. Intrusion resilient can be effective only if combined with traditional measures to avoid and remove vulnerabilities in system components, and with intrusion detection mechanisms to help respond to attacks before it is too late.

\section{References}

1. Hung-Yu Chien, New spproach to authorization and authentication in distributed environments, Communications of the CCISA, vol.9 no.3, pp.63-69, Jun. 2003

2. Mambo, M., Usuda, K., and Okamoto, E., Proxy signatures for delegating signing operation, Proc. 3rd ACM conf. on Computer and Communications Security, 1996.

3. P. Feldman, A Practical Scheme for Non-interactive Verifiable Secret Sharing, Proc. 28th IEEE Annual Symposium on Foundations of Computer Science, pp. 427-437, Oct. 1987

4. A. Shamir. How to Share a Secret. Communications of the ACM, vol. 22, no. 11, pp. 612613, Nov. 1979.

5. Chaum D. and Pedersen. T. Wallet Databases with Observers. In Crypto' 92 , number 740 in Lecture Notes in Computer Science, Santa Barbara, CA, Springer-Verlag, 1992, pp. 89-105

6. Yair Frankel, Peter Gemmell, Philip D. MacKenzie, and Moti Yung. Optimal resilience proactive publickey cryptosystems. In IEEE Symposium on Foundations of Computer Science, pp 384-393, 1997.

7. Yair Frankel, Peter Gemmell, Phillip D MacKenzie, and Moti Yung. Proactive RSA. In Advanced In Cryptology - Crypto 97, volume 1294 of Lecture Notes in Computer Science, pp 440-454 Springer-Verlag, 1997.

8. Globus OGSA Home Page. http://www.globus.org/ogsa/

9. Foster I, Kesselman C. The Grid: Blueprint for a new computing infrastructure. San Fransisco: Morgan Kaufmann Publisher, 1999. 